\title{
Efeito do treinamento aquático no perfil lipídico e na hipertrofia ventricular esquerda de camundongos hiperlipidêmicos
}

\author{
Effect of water training on the lipid \\ profile and left ventricular hypertrophy in \\ hyperlipidemic mice
}

Rev Bras Ativ Fis Saúde p. 453-463 DOI:

http://dx.doi.org/10.12820/rbafs.v.18n4p453

1 UNIFENAS- Mestre - Docente dos Cursos de Educação Física e Nutrição - Alfenas/MG 2 UNIFENAS- Curso de Educação Física Alfenas/MG

3 UNICAMP - Doutorando em Biologia Funcional e Molecular - Campinas/SP

4 UNIFENAS - Livre Docente - Curso de Educação Física - Alfenas/MG Faculdade de Ciências da Universidade Estadual Paulista Júlio de Mesquita Filho (UNESP); Programa de Mestrado/Doutorado em Promoção de Saúde da Universidade de Franca; Faculdade de Medicina da Universidade de Ribeirão Preto.

Bruno Barbosa Rosa

Leandro dos Santos 3

Cassiano Merussi Neiva ${ }^{4}$

José Antonio Dias Garcia ${ }^{5}$

5 UNIFENAS- Doutor - Docente do Curso de Medicina -Alfenas/MG

\section{Resumo}

O objetivo do presente estudo foi analisar os efeitos do treinamento aquático (TA) no perfil lipídico e nas estruturas ventriculares esquerdas de camundongos hiperlipidêmicos. Foram utilizados 28 camundongos LDLr-/-, machos, divididos aleatoriamente em 4 grupos: sedentários alimentados com ração padrão $(\mathrm{C})$; treinados alimentados com ração padrão (C+TRE), sedentários alimentados com ração hiperlipídica (HL) e treinados alimentados com ração hiperlipídica (HL+TRE). Os animais dos grupos treinados praticaram TA em intensidade moderada, por 60 minutos durante 60 dias consecutivos. Após 48 horas do término do período de treinamento e 12 horas de jejum de repouso os animais foram eutanasiados, o sangue coletado e o soro utilizado para análises laboratoriais de triglicérides, colesterol total e suas frações (LDL, HDL, VLDL). O coração foi removido e o ventrículo esquerdo foi pesado a fresco para se calcular a proporção do peso ventricular esquerdo $(\mathrm{mg}) /$ peso do animal $(\mathrm{g})$. Os resultados mostraram que o TA sem cargas adicionais foi mais eficiente na melhora das concentrações plasmáticas de lipídeos quando acompanhado por uma dieta adequada, confirmando que a associação exercício físico com dieta equilibrada é essencial para obtenção de seus benefícios. $O$ treinamento ocasionou hipertrofia ventricular esquerda excêntrica no grupo alimentado com dieta padrão e, principalmente, diminuiu o depósito de colágeno intersticial no miocárdio dos animais alimentados com dieta hiperlipídica, o que pode indicar uma melhora na função diastólica com consequente melhora da função sistólica. Concluiu-se que o treinamento aquático de intensidade leve a moderada induz adaptações benéficas e profiláticas ao coração, promovendo saúde e prevenindo doenças.

\section{Palavras-chave}

Hipertrofia ventricular; Natação,;Dislipidemias.

\begin{abstract}
This study assessed the effects of Water training on the lipid profile and left ventricular structures in hyperlipidemic mice. Twenty-eight male LDLr-/- mice were randomly separated into 4 groups: sedentary, fed a standard diet (C); exercising, fed a standard diet (C+TRE); sedentary, fed a byperlipidic diet (C+HL); and exercising, fed a hyperlipidic diet $(E+H L)$. The exercising mice trained daily for 60 minutes during 60 days. After 48 hours of the end of the training period and 12 hours of rest fasting the animals were underwent euthanasia and the blood was collected for measuring the plasma levels of triglycerides, total cholesterol and its fractions ( $L D L, H D L, V L D L)$. The heart was removed and the left ventricle was weighed fresh to calculate the ratio left-ventricle weight $(\mathrm{mg}) /$ body weight $(\mathrm{g})$. The results showed that the training was more effective in improving lipid plasma levels when combined with a balanced diet, thereby confirming that it is essential to associate physical exercise and diet. The training protocol resulted in eccentric left ventricular hypertrophy in the standard-diet group and decreased interstitial collagen deposition in the myocardium of the high-fat-diet animals, which may indicate an improved diastolic function with consequent improvement in the systolic function. It was concluded that regular moderate aerobic exercise induce beneficial and prophylactic adaptations to heart which promoted a better health condition and prevention to diseases.
\end{abstract}

\section{Keywords}

Ventricular hypertrophy; Water Training; Dyslipidemia. 


\section{INTRODUÇÃO}

A hipertrofia cardíaca representa um processo adaptativo importante. É um eficiente mecanismo de compensação quando o músculo é exposto à sobrecargas crônicas de trabalho ${ }^{1,2}$, observadas em condições patológicas, como em doenças cardiovasculares, e também fisiológicas, durante exercícios físicos regulares. Decorre do aumento das dimensões dos cardiomiócitos com proliferação de tecido conjuntivo intersticial, dependendo da intensidade do processo hipertrófico ${ }^{3}$.

Os padrões de remodelamento cardíaco são determinados pelas sobrecargas de pressão ou de volume, podendo ser classificadas em excêntricas ou concêntricas. Diferenciam-se principalmente pelas concentrações de vasos sanguíneos e matriz intersticial colágena, diâmetro da cavidade ventricular, adição de sarcômeros em série ou em paralelo ${ }^{4}$ e espessura parietal relativa $(E P R)^{5}$ que representa a relação da espessura parietal/raio da cavidade ventricular esquerda.

Nas hipertrofias concêntricas há aumento da massa ventricular, da EPR, pela inserção de sarcômeros em paralelo, redução dos diâmetros cavitários e proliferação da matriz intersticial seguida de fibrose que, em conjunto, levam à disfunção sistólica e diastólica ${ }^{5}$. Tal condição surge em decorrência de um aumento da resistência à ejeção ou à sobrecarga de pressão, observados em condições patológicas, como na hipertensão arterial, por exemplo ${ }^{1}$. É uma condição que compromete o estado de saúde do indivíduo ${ }^{1,3}$.

No entanto, a hipertrofia cardíaca excêntrica caracteriza-se pelo crescimento do cardiomiócito com a inserção de sarcômeros em série e poucas alterações da matriz intersticial. Ocorre um aumento da massa ventricular com manutenção ou aumento dos diâmetros cavitários ${ }^{1}$ e EPR normal ${ }^{5}$. Surge em decorrência da sobrecarga de volume, que pode ser observada durante a prática de exercícios aeróbios ${ }^{1}$ contínuos de intensidade moderada à intensa, onde o estiramento muscular imposto favorece a degradação do colágeno, favorecendo a função diastólica ${ }^{4}$.

Portanto, a hipertrofia cardíaca excêntrica é uma das principais adaptações fisiológicas ao treinamento aeróbio, sendo a intensidade do exercício fator determinante. Seu desenvolvimento traz um impacto importante na qualidade de vida dos indivíduos ${ }^{2}$. Por outro lado, ainda não é totalmente claro o papel do exercício físico aeróbio no tratamento da hipertrofia concêntrica já instalada.

Por outro lado, o uso habitual de um padrão dietético rico em gordura, é apontado como potencializador da HVE, uma vez que, alterando a concentração de lipídios plasmáticos, favorece as condições ao desenvolvimento de tal dismorfismo ${ }^{8}$. Em estudos anteriores ${ }^{3,8}$, os pesquisadores apontam a relação entre dietas hiperlipídicas e danos vasculares e no miocárdio de animais experimentais submetidos a padrão dietético gorduroso. Nesse sentido ainda, estudos da última década ${ }^{6,7}$, demonstraram uma efetiva capacidade no desenvolvimento de dislipidemias e lesões vasculares avançadas em camundongos transgênicos (apoE knockout mouse), que compartilham aspectos semelhantes às lesões humanas avançadas, especialmente quando submetidos a dietas hiperlipídicas.

Face ao exposto o objetivo deste estudo foi analisar os efeitos do treinamento aquático de intensidade aeróbia no perfil lipídico e nas estruturas ventriculares esquerdas de camundongos hiperlipidêmicos.

\section{MÉTODOS}

Para o estudo, foram empregados camundongos homozigotos para a ausência do 
gene do receptor de LDL (LDLr-/-) gerados no background C57BL6, machos com 3 meses de idade, pesando inicialmente as $23 \pm 3 \mathrm{~g}$, os quais foram pesados semanalmente durante todo o estudo em balança eletrônica de precisão. Estes camundongos transgênicos são capazes de desenvolver lesões vasculares avançadas que compartilham aspectos semelhantes às lesões humanas avançadas ${ }^{14,15}$. Os animais foram obtidos do Jackson Laboratories (EUA) e criados em um biotério, com controle de temperatura e de ciclo claro/escuro (12 horas). Foram utilizados 28 camundongos, divididos aleatoriamente em 4 grupos experimentais. A Tabela 1 apresenta a divisão e denominação dos grupos bem como a característica dos protocolos alimentares e de treinamento empregados em cada grupo:

Tabela 1 - Grupos experimentais e seus respectivos protocolos.

\begin{tabular}{|c|c|c|}
\hline Grupos & Protocolo animal & Denominação \\
\hline 1 & $\begin{array}{l}\text { Camundongos LDLr-/- alimentados com ração padrão para } \\
\text { roedores (Nuvital@) por } 75 \text { dias e permaneceram sedentários. }\end{array}$ & C \\
\hline 2 & $\begin{array}{l}\text { Camundongos LDLr-/- alimentados com ração padrão para } \\
\text { roedores (Nuvital }(\text { ) por } 75 \text { dias. Após os primeiros } 15 \text { dias } \\
\text { de experimento foram submetidos a } 3 \text { sessões semanais de } \\
\text { treinamento aquático, em dias alternados, por } 60 \text { minutos, } \\
\text { sem uso de carga adicional além do próprio peso corpóreo. }\end{array}$ & $C+T R E$ \\
\hline 3 & $\begin{array}{l}\text { Camundongos LDLr-/- alimentados com ração hiperlipídica } \\
\text { ( } 20 \% \text { de gordura total, 1,25\% de colesterol, 0,5 \% ácido } \\
\text { cólico) por } 75 \text { dias e permaneceram sedentários. }\end{array}$ & $\mathrm{HL}$ \\
\hline 4 & $\begin{array}{l}\text { Camundongos LDLr-/- alimentados com ração hiperlipídica } \\
\text { por } 75 \text { dias. Após os primeiros } 15 \text { dias de experimento foram } \\
\text { submetidos à } 3 \text { sessões semanais de treinamento aquático, } \\
\text { em dias alternados, por } 60 \text { minutos, sem uso de carga } \\
\text { adicional além do próprio peso corpóreo. }\end{array}$ & $\mathrm{HL}+\mathrm{TRE}$ \\
\hline
\end{tabular}

Inicialmente os animais receberam suas respectivas rações: padrão (grupos $\mathrm{C}$ e C+TRE) ou hiperlipídica (HL e HL+TRE), por 15 dias consecutivos para que, conforme Garcia e Incerpi ${ }^{6}$ houvesse desenvolvimento de HVE nos grupos alimentados com a ração hiperlipídica.

Os animais dos grupos treinados foram submetidos ao treinamento aquático em tanques individuais cúbicos com as dimensões de $30 \mathrm{~cm}$ de altura por $15 \mathrm{~cm}$ de lados totalizando $675 \mathrm{~cm}^{3}$ (Figura 1), com água ao nível de $25 \mathrm{~cm}$ de altura, equivalente a 5,625 litros, suficiente para que os animais não se apoiassem ao fundo durante as sessões de treinamento. A temperatura da água foi mantida entre 32$36^{\circ} \mathrm{C}$. As sessões de treinamento iniciaram após os primeiros 15 dias, utilizados para o desenvolvimento da $\mathrm{HVE}^{6}$, e partir de então ocorreram durante 8 semanas subsequentes, com frequência de 3 vezes/semana, em dias alternados, sempre no mesmo horário. Os animais eram colocados e retirados da água, pela cauda, delicadamente, sempre pelo mesmo pesquisador, responsável pela condução da lida dos animais durante todo o estudo. Os animais realizaram as sessões de treinamento sem a adição de quaisquer cargas adicionais aos seus corpos. $\mathrm{O}$ tempo de $60 \mathrm{mi}-$ nutos consecutivos em atividade contínua na água foi garantido por observação e intervenção do pesquisador durante todas as sessões de treinamento.

Os animais dos grupos sedentários (C, HL), foram também submetidos ao mesmo ambiente aquático, por 5 minutos apenas, nos mesmos dias de treinamento dos grupos treinados, seguindo-se os mesmos critérios dos grupos treinados, a fim de mimetizar o estresse aquático ${ }^{16}$. Ao final do período de 75 dias de treinamento 
e após 48 horas de intervalo da última sessão de treino, para evitar interferências agudas, respeitando o ciclo circadiano dos animais, os mesmos foram submetidos a 12 horas de jejum prévio em repouso, sendo então pesados e anestesiados antes da eutanásia. O sangue foi coletado por punção do plexo venoso retro-orbital, utilizando capilares heparinizados. Após separação por centrifugação imediata a 3000 RPM por 10 minutos, o soro foi encaminhado para análises laboratoriais de triglicérides, colesterol total e suas frações (LDL, HDL, VLDL). Os valores das concentrações do colesterol VLDL foram obtidos pela divisão dos valores de triglicérides por 5 e os valores do colesterol LDL pela subtração de colesterol HDL e VLDL do colesterol total ${ }^{17}$.

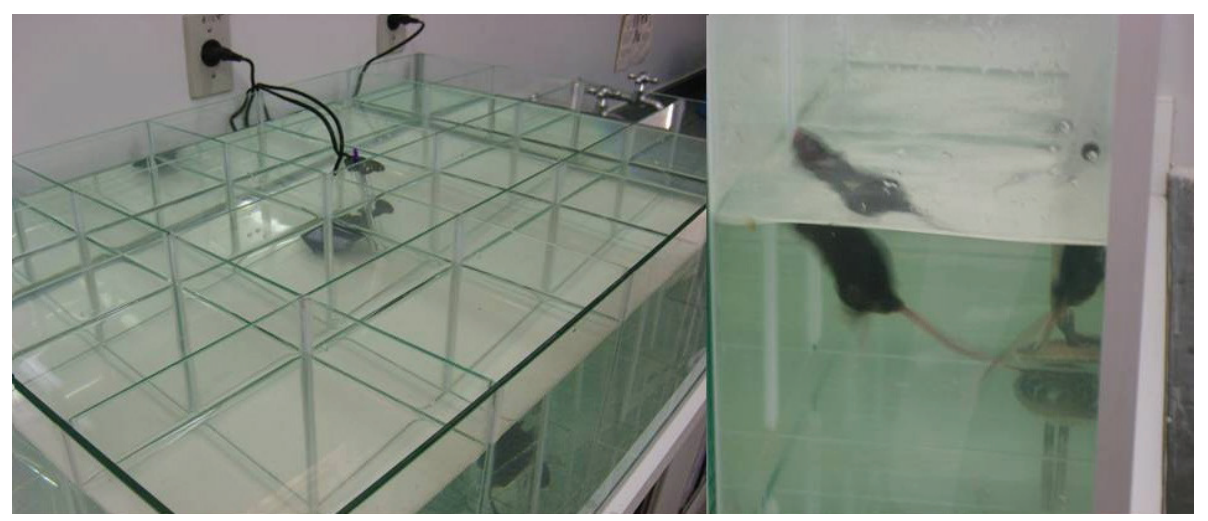

Figura 1 - Tanque utilizado para sessões de treinamento aquático.

Após a toracotomia foram removidos os átrios e a parede livre do ventrículo direito permanecendo somente o ventrículo esquerdo (VE), que foi pesado a fresco (mg), calculando-se a proporção do peso ventricular esquerdo $(\mathrm{mg}) /$ peso do animal $(\mathrm{g})^{6}$.

Os VE foram fixados em formalina $10 \%$ por 48 horas e incluídos em parafina para cortes histológicos de $4 \mu \mathrm{m}$ de espessura. Posteriormente foram corados com hematoxilina/eosina e picrosírius red, para análises morfológica e qualitativa de colágeno por meio de luz polarizada. As imagens foram capturadas através do programa Laudo e Imagem Net e salvas em um formato digital que permite a visualização das mesmas como foto na tela do computador (formato JPEG) permitindo assim posteriores análises detalhadas para medida do diâmetro dos cardiomiócitos, da espessura parietal, área da câmara cardíaca e quantificação da área de colágeno no tecido cardíaco seguindo critério padrão ${ }^{8,18}$. A seguir foi calculada a EPR ${ }^{8}$.

Após prévia análise descritiva, valores preliminares foram submetidos à análise de normalidade de sua distribuição através de teste de Kolmogorov-Smirnov. Os dados finais foram expressos como média \pm desvio padrão (DP) e de acordo com a presença normalidade apresentada foi empregada à análise de variância (ANOVA) seguida de teste de Tukey para comparação dos diferentes grupos. As diferenças foram consideradas significativas quando o valor de "p" $<0,05$.

Todos os procedimentos utilizados foram efetivamente descritos e explicados no projeto prévio apresentado ao CEP de Experimentação Animal da Universidade o qual foi aprovado ${ }^{\circ}$ do protocolo: 10 A/2009.

\section{RESULTADOS}

Os resultados apresentados na TABELA 2 demonstram que a concentração de $\mathrm{CT}$ diminuiu nos camundongos do grupo $\mathrm{C}+\mathrm{TRE}$ quando comparadas às do 
grupo $\mathrm{C}(\mathrm{p}<0,01)$. Os camundongos dos grupos HL e HL+TRE apresentaram hipercolesterolemia severa quando comparados com os dos grupos $\mathrm{C}$ e $\mathrm{C}+\mathrm{TRE}$, porém as concentrações plasmáticas de $\mathrm{CT}$ não foram diferentes entre os grupos HL e HL+TRE.

As concentrações plasmáticas de HDL foram menores nos camundongos do grupo HL ( $\mathrm{p}<0,01)$ quando comparadas com as dos grupos $\mathrm{C}, \mathrm{C}+\mathrm{TRE}$ e HL+TRE.

Nos camundongos do grupo C+TRE as concentrações plasmáticas de LDL diminuíram quando comparadas as do grupo $\mathrm{C}(\mathrm{p}<0,03)$. Os camundongos dos grupos HL e HL+TRE apresentaram aumento severo de LDL quando comparados com os dos grupos C e C+TRE, porém as concentrações plasmáticas de LDL não foram diferentes entre eles.

Com relação às concentrações plasmáticas de VLDL, verificou-se diferenças entres os 4 grupos experimentais, sendo que os grupos C+TRE e HL+TRE apresentaram diminuição do VLDL $(\mathrm{p}<0,05)$ quando comparados com os do grupo $\mathrm{C}$ e HL, respectivamente.

Diferenças nas concentrações plasmáticas de TG foram verificadas nos 4 grupos experimentais, sendo que os grupos C+TRE e HL+TRE apresentaram diminuição do TG quando comparados com os do grupo $\mathrm{C}$ e HL, respectivamente.

Tabela 2 - Concentrações plasmáticas de CT, HDL, LDL, VLDL e TG dos animais alimentados com dieta padrão (C), alimentados com dieta padrão e submetidos à treinamento aquático (C+TRE), alimentados com dieta hiperlipídica $(\mathrm{HL})$ e alimentados com dieta hiperlipídica e submetidos à treinamento aquático (HL+TRE).

\begin{tabular}{|c|c|c|c|c|}
\hline $\begin{array}{l}\text { Níveis Plasmáticos } \\
\text { de Lipídeos } \\
(\mathrm{mg} / \mathrm{dL})\end{array}$ & C & $\mathrm{C}+\mathrm{TRE}$ & $\mathrm{HL}$ & $\mathrm{HL}+\mathrm{TRE}$ \\
\hline $\mathrm{N}$ & 7 & 7 & 7 & 7 \\
\hline $\mathrm{CT}$ & $\begin{array}{l}356 \pm 21^{a} \\
(p<0,01)\end{array}$ & $\begin{array}{l}281 \pm 14^{b} \\
(p<0,01)\end{array}$ & $563 \pm 32^{c}$ & $584 \pm 41^{c}$ \\
\hline $\mathrm{HDL}$ & $59 \pm 4^{a}$ & $68 \pm 5^{a}$ & $\begin{array}{c}26 \pm 2^{b} \\
(p<0,01)\end{array}$ & $67 \pm 4^{a}$ \\
\hline LDL & $\begin{array}{l}272 \pm 18^{a} \\
(p<0,03)\end{array}$ & $\begin{array}{l}195 \pm 16^{b} \\
(p<0,03)\end{array}$ & $461 \pm 31^{c}$ & $467 \pm 36^{c}$ \\
\hline VLDL & $\begin{array}{c}25 \pm 1^{a} \\
(p<0,05)\end{array}$ & $\begin{array}{c}18 \pm 2^{b} \\
(p<0,05)\end{array}$ & $\begin{array}{c}76 \pm 6^{c} \\
(p<0,05)\end{array}$ & $50 \pm 3^{d}$ \\
\hline TG & $\begin{array}{l}125 \pm 13^{a} \\
(p<0,05) \\
(p<0,03)\end{array}$ & $\begin{array}{c}90 \pm 7^{b} \\
(p<0,03)\end{array}$ & $\begin{array}{l}380 \pm 26^{c} \\
(p<0,05)\end{array}$ & $250 \pm 22^{d}$ \\
\hline
\end{tabular}

Os valores foram expressos como média \pm desvio padrão; $\mathrm{CT}=$ Colesterol Total, $\mathrm{HDL}=$ colesterol de alta densidade), $\mathrm{LDL}=$ colesterol de baixa densidade, $\mathrm{VLDL}=$ colesterol de muito baixa densidade, TG = triglicérides. Letras iguais indicam não significância e letras diferentes indicam significância. Valore de " $\mathrm{p}$ " abaixo das médias é relativo a subsequente letra diferente. Anova complementado por Tukey.

$\mathrm{Na}$ análise da proporção do peso ventricular esquerdo $(\mathrm{mg}) /$ peso do animal (g) foi observada HVE nos grupos C+TRE, HL e HL+TRE quando comparados com o grupo C. Entretanto, no grupo HL+TRE a hipertrofia foi mais significativa, diferenciando-a dos animais dos grupos C+TRE e HL. Esse resultado expressa as alterações morfométricas encontradas, mostrando aumento nos diâme- 
tros dos cardiomiócitos nos camundongos dos grupos C+TRE, HL e HL+TRE e da espessura parietal ventricular esquerda nos camundongos dos grupos HL e HL+TRE quando comparados com os do grupo C. Entretanto, no grupo HL+TRE esses parâmetros foram mais significativos, diferenciando-os dos animais dos grupos C+TRE e HL. Com relação ao depósito de colágeno intersticial do miocárdio, observou-se que houve um maior depósito nos camundongos do grupo HL (Tabela 3).

$\mathrm{Na}$ avaliação da dimensão da cavidade ventricular esquerda observou-se que, nos grupos HL e HL+TRE, houve uma diminuição significativa da cavidade ventricular ao comparar-se com os grupos $\mathrm{C}$ e C+TRE (Tabela 3).

Os dados da EPR mostraram que os animais dos grupos $\mathrm{C}$ e $\mathrm{C}+\mathrm{TRE}$ apresentaram valores iguais, enquanto que os valores encontrados para os grupos $\mathrm{HL}$ e HL+TRE foram superiores, diferenciando-os dos animais grupos $\mathrm{C}$ e $\mathrm{C}+\mathrm{TRE}$. Ao comparar-se o grupo HL com HL+TRE, observou-se que a EPR foi superior no grupo HL+TRE.

Tabela 3 - Parâmetros morfométricos dos animais alimentados com dieta padrão (C), alimentados com dieta padrão e submetidos à treinamento aquático ( $C+T R E)$, alimentados com dieta hiperlipídica $(\mathrm{HL})$ e alimentados com dieta hiperlipídica e submetidos à treinamento aquático (HL + TRE).

\begin{tabular}{|c|c|c|c|c|}
\hline $\begin{array}{c}\text { Parâmetros } \\
\text { morfométricos }\end{array}$ & C & $C+$ TRE & $\mathrm{HL}$ & $\mathrm{HL}+\mathrm{TRE}$ \\
\hline $\mathrm{N}$ & 7 & 7 & 7 & 7 \\
\hline $\begin{array}{l}\text { Peso do VE /peso } \\
\text { animal }\end{array}$ & $\begin{array}{l}3,4 \pm 0,3^{a} \\
(p<0,05)\end{array}$ & $\begin{array}{l}3,7 \pm 0,3^{b} \\
(p<0,05)\end{array}$ & $\begin{array}{l}3,7 \pm 0,2^{b} \\
(p<0,05)\end{array}$ & $4,1 \pm 0,3^{c}$ \\
\hline $\begin{array}{l}\text { Diâmetro de } \\
\text { cardiomiócito }\end{array}$ & $\begin{array}{c}11,7 \pm 0,1^{a} \\
(p<0,05)\end{array}$ & $\begin{array}{l}14 \pm 0,2^{b} \\
(p<0,05)\end{array}$ & $\begin{array}{l}13,2 \pm 0,2^{b} \\
(p<0,05)\end{array}$ & $15,9 \pm 0,2^{c}$ \\
\hline $\begin{array}{l}\text { Espessura parietal } \\
\text { ventricular esquerda }\end{array}$ & $\begin{array}{l}59,5 \pm 0,4^{a} \\
(p<0,03)\end{array}$ & $\begin{array}{l}58,5 \pm 5^{a} \\
(p<0,03)\end{array}$ & $\begin{array}{c}75 \pm 5^{b} \\
(p<0,05)\end{array}$ & $85 \pm 8^{c}$ \\
\hline $\begin{array}{l}\text { Dimensão da cavidade } \\
\text { ventricular esquerda } \\
\left(\mu \mathrm{m}^{2}\right)\end{array}$ & $\begin{array}{c}3419,3 \pm 65^{a} \\
(p<0,001)\end{array}$ & $\begin{array}{c}3401,3 \pm 63^{a} \\
(p<0,001)\end{array}$ & $1452,9 \pm 42^{b}$ & $1245,1 \pm 59^{b}$ \\
\hline $\begin{array}{l}\text { Espessura parietal } \\
\text { relativa }\end{array}$ & $\begin{array}{c}0,017 \pm 0,1^{a} \\
(p<0,01)\end{array}$ & $\begin{array}{l}0,017 \pm 0,1^{\mathrm{a}} \\
(p<0,01)\end{array}$ & $\begin{array}{c}0,051 \pm 0,4^{b} \\
(p<0,05)\end{array}$ & $0,068 \pm 0,3^{c}$ \\
\hline
\end{tabular}

Os valores foram expressos como média \pm desvio padrão. Letras iguais indicam não significância e letras diferentes indicam significância. Valore de " $\mathrm{p}$ " abaixo das médias é relativo a subsequente letra diferente. Anova complementado por Tukey.

\section{DISCUSSÃO}

Apesar de os animais utilizados neste estudo apresentarem hipercolesterolemia espontânea, ainda assim, pode-se observar que o treinamento aquático associado à dieta padrão foi eficiente no controle das concentrações plasmáticas de lipídeos. Contudo, ao analisar os grupos que foram alimentados com dieta hiperlipídica, percebeu-se uma elevação significativa das concentrações plasmáticas de CT e LDL, ou seja, apresentaram hipercolesterolemia severa, sugerindo que o treinamento aquático de carga leve a moderada, com o próprio peso do corpo e sem cargas adicionais, associada a uma dieta hiperlipídica não foi eficiente na melhora destes parâmetros. Entretanto, na observação dos valores de VLDL e TG, também 
aumentados nos grupos alimentados com dieta hiperlipídica, o treinamento aquático de carga leve a moderada foi eficiente em sua redução.

Ao analisar as concentrações plasmáticas de HDL demonstrados neste estudo, pode-se observar que os valores encontrados para os camundongos do grupo HL foram menores $(\mathrm{p}<0,01)$, demonstrando que o treinamento aquático de carga leve a moderada, apesar de não ter contribuído na melhora das concentrações de CT e LDL dos animais alimentados com dieta hiperlipídica, foi eficiente na elevação plasmática de HDL.

Halle et al. ${ }^{20}$ demonstraram que quanto melhor a aptidão física e menor o índice de massa corporal, há uma tendência de que as concentrações de lipídeos sanguíneos sejam favoráveis. Pitanga ${ }^{19}$ encontrou correlações estatisticamente significativas entre a prática de exercícios físicos e as concentrações de HDL. Em seu estudo, os indivíduos ativos apresentaram maiores concentrações de HDL em comparação aos sedentários. Resultados inversos foram observados em relação à LDL, confirmando os achados do presente estudo.

Kraus et al. ${ }^{10}$ estudaram indivíduos sedentários, com sobrepeso ou obesos com moderada dislipidemia em três grupos de exercício e um controle, os quais variaram em intensidade ( 40 a $80 \% \mathrm{VO}_{2}$ max - volume máximo de oxigênio absorvido) e/ou volume (19,2 a $32 \mathrm{~km} /$ semana). Os resultados demonstraram grande associação entre o volume do exercício físico e melhora no perfil lipoprotéico.

Donovan et al..$^{22}$ observaram que a intensidade do exercício tem maior relação com a redução dos fatores de risco para doenças cardíacas. Foi observado que, após 24 semanas de exercício físico a $50 \%$ ou a $80 \% \mathrm{VO}_{2}$ max, o grupo de alta intensidade apresentou significativo aumento no $\mathrm{VO}_{2}$ max e redução nas concentrações de LDL e CT. Em nosso estudo também foi possível observar reduções significativas de LDL e CT no grupo ativo alimentado com dieta padrão. Contudo, no grupo ativo alimentado com dieta hiperlipídica, não houve diferença significativa, reafirmando a importância da dieta equilibrada associada à prática do exercício físico.

Leon e Sanches ${ }^{23}$ avaliaram artigos a respeito das alterações lipídicas decorrentes do exercício físico aeróbio isolado ou combinado com dieta, e observaram que a redução nas concentrações de LDL, CT e TG é menos frequente em comparação ao aumento de HDL, em estudos com período igual ou superior a 12 semanas de intervenção. As concentrações de HDL aumentaram significativamente em 47\% dos estudos. Já em nossa pesquisa, as concentrações de HDL dos grupos treinados foram estatisticamente iguais às do grupo sedentário alimentado com dieta padrão (C) e maiores que os do grupo HL (sedentário alimentado com dieta hiperlipídica). Este dado pode indicar que o exercício físico aeróbio de carga leve a moderada, com frequência de 3 vezes/semana, pode se mostrar interessante para indivíduos que têm uma dieta rica em lipídios e sejam fisicamente ativos.

Prado e Dantas ${ }^{11}$ mencionaram como modelo de exercício físico aeróbio, intensidade moderada (50\% a 70\% do $\mathrm{VO}_{2} \max$ ), com duração mínima de 30 minutos, pelo menos três sessões semanais. Os autores acreditam que este modelo pode ser ideal para induzir modificações no perfil lipídico basal em indivíduos de diferentes faixas etárias e níveis lipídicos, salientando que a associação da dieta adequada e da perda de massa corporal parece ser fundamental. No presente estudo, a relação da dieta com o exercício também se mostrou importante.

Alguns estudos de Rique et al. ${ }^{24}$ sugeriram que o exercício aeróbio reduz de forma consistente as concentrações plasmáticas dos triglicérides, embora a diminuição do colesterol total e LDL seja controversa, pois é mais eficiente quando associada à perda de peso e à restrição energética. Na presente pesquisa a redução 
nas concentrações de TG também foi observada nos grupos ativos.

Um estudo realizado por Bernardes et al..$^{25}$ com ratos Wistar visou verificar os efeitos da dieta hiperlipídica e do treinamento por natação no metabolismo. Foi observado que a dieta hiperlipídica aumentou significativamente a concentração plasmática de triglicérides, contudo no grupo TH (treinados e alimentados com dieta hiperlipídica) houve diminuição na concentração de triglicérides em 1 hora de recuperação, em relação ao repouso, confirmando os dados encontrados no presente estudo.

No estudo de Bernardes et al. ${ }^{25}$ a dieta hiperlipídica também aumentou a concentração de colesterol total. Além disso, os animais treinados exibiram maiores valores para esta variável em repouso e em uma hora de recuperação, em relação aos sedentários. O grupo TN (treinados e alimentados com dieta normocalórica) apresentou diminuição significativa desta variável quando avaliado em 2 horas de recuperação, comparando-se ao repouso, dados que corroboram os encontrados no presente estudo.

Ainda neste estudo, os resultados encontrados por Bernardes et al..$^{25}$ para o HDL mostraram que o treinamento moderado de natação e o consumo de dieta hiperlipídica interagiram promovendo os maiores valores desta variável no grupo TH (treinados alimentados com dieta hiperlipídica). Resultados semelhantes foram também encontrados no presente estudo.

A maior concentração de HDL pode estar relacionada ao maior conteúdo de gordura da dieta e ao fato de que o exercício, realizado de maneira regular, tem promovido aumento desta variável em humanos ${ }^{25}$.

No caso do CT, parece que o treinamento acentuou o efeito da dieta em aumentar sua concentração. De fato, estudos anteriores, evidenciaram que a constante utilização de gordura como substrato energético pode acelerar a sua biossíntese. Da mesma forma, treinamento e dieta se associaram, promovendo os maiores valores para fração HDL. Sugeriu-se que os efeitos benéficos do treinamento em promover uma rápida recuperação dos substratos endógenos, principalmente carboidratos, são prejudicados pela dieta hiperlipídica, quando esta é associada ao treinamento. Segundo Bernardes et al. ${ }^{25}$ estes resultados podem estar evidenciando o desenvolvimento de um mecanismo de defesa do animal, que tenta estocar primeiramente energia na forma de substrato lipídico, para ser utilizado em futuras sessões de exercício.

Com relação aos parâmetros morfológicos e morfométricos analisados neste estudo, a presença de hipertrofia ventricular nos camundongos dos grupos $\mathrm{C}_{+}-$ TRE, HL e HL+TRE foi evidente. Entretanto, o tipo de hipertrofia foi diferente entre os grupos alimentados com dieta padrão e os alimentados com dieta hiperlipídica.

O grupo $\mathrm{C}+\mathrm{TRE}$ apresentou elevação da proporção peso do $\mathrm{VE} /$ peso do animal e aumento do diâmetro dos cardiomiócitos, indicando hipertrofia de ventrículo esquerdo. Ao se observar os dados referentes à espessura parietal ventricular esquerda, à dimensão da cavidade ventricular esquerda e ao depósito de colágeno não houve diferenças significativas ao compararmos com o grupo C. Estes dados, aliados ao valor encontrado na espessura parietal relativa, sugerem que esta hipertrofia foi excêntrica, reforçando achados de outros autores que afirmam que o exercício aeróbio induz hipertrofia cardíaca excêntrica ${ }^{27}$.

Ao comparar os grupos C+TRE com HL percebemos valores semelhantes para proporção peso do VE/peso do animal e para diâmetro dos cardiomiócitos, mostrando que o treinamento aquático induziu hipertrofia no grupo $\mathrm{C}+\mathrm{TRE}$ (ca- 
mundongos saudáveis) e que a dieta hiperlipídica induziu hipertrofia no grupo HL (camundongos hiperlipidêmicos). Contudo, ao analisar os demais parâmetros, fica claro que os padrões de hipertrofia dos dois grupos são diferentes. No grupo HL houve significativa elevação da espessura parietal ventricular esquerda com concomitante diminuição da dimensão da cavidade ventricular esquerda, sugerindo adição de sarcômeros em paralelo, característica da hipertrofia concêntrica ${ }^{8,9} \mathrm{O}$ valor encontrado para a espessura parietal relativa e o maior depósito de colágeno no espaço intersticial confirmam os dados sugestivos de HVE concêntrica.

A maioria das características encontradas nos camundongos do grupo HL foi também encontrada nos camundongos do grupo HL+TRE, entretanto com valores mais expressivos. Isso indica que a associação da dieta hiperlipídica com o treinamento aquático, com carga leve a moderada, não foi eficiente para reverter ou estagnar o desenvolvimento do padrão concêntrico de hipertrofia. Contudo, um dado relevante a ser considerado é o do depósito de colágeno no espaço intersticial. O grupo HL+TRE apresentou características parecidas com as dos camundongos dos grupos $\mathrm{C}$ e $\mathrm{C}+\mathrm{TRE}$. Portanto, apesar de a hipertrofia encontrada nos animais do grupo HL+TRE apresentar indícios significativos para ser classificada como concêntrica, o treinamento aquático conseguiu impedir um depósito excessivo de colágeno, o que, provavelmente, melhorou a função cardíaca ${ }^{8}$.

Ghorayeb et al. ${ }^{27}$ afirmaram que a hipertrofia induzida pelo exercício não se acompanha de fibrose e outras alterações estruturais, observadas em condições patológicas, pois não induz à desproporção entre oferta e consumo de oxigênio, mesmo em condições de esforço máximo, confirmando os achados da presente pesquisa, nos grupos C+TRE e HL+TRE.

Pluim et a ${ }^{28}$ demonstraram que as alterações da massa ventricular esquerda e do tamanho da cavidade ventricular com o treinamento e condicionamento físico ocorrem em paralelo com alterações do $\mathrm{VO}_{2} \max$, sugerindo, fortemente, que a hipertrofia ventricular excêntrica se associa com melhor função cardíaca.

Dados relatados por Ghorayeb et al. ${ }^{27}$ mostraram que maratonistas com hipertrofia ventricular esquerda, com excelente nível de treinamento, apresentam função ventricular esquerda normal, tanto sistólica como diastólica, consumo máximo de oxigênio elevado e não apresentam respostas anômalas ao teste ergométrico. Natali ${ }^{26}$ afirma que o aumento na contratilidade intrínseca dos miócitos é um mecanismo potencial para a explicação da melhora da função contrátil do miocárdio induzida pelo exercício.

Pluim et al. ${ }^{28}$ observaram em atletas praticantes de esportes de resistência e força, que os diversos índices de contratilidade não invasivos utilizados para avaliar a função ventricular esquerda foram normais, apesar do aumento acentuado da cavidade e espessura ventriculares. Outro estudo ${ }^{28}$ avaliou a dimensão ecocardiográfica da cavidade ventricular esquerda. Consideraram que a cavidade ventricular esquerda estava substancialmente aumentada em $14 \%$ dos atletas. Todos tinham função ventricular esquerda sistólica global dentro dos limites da normalidade, e após seguimento de 12 anos, os atletas permaneceram assintomáticos e não apresentaram anomalia da performance cardíaca. Os principais determinantes da dimensão da cavidade ventricular esquerda foram a maior superfície corpórea e a participação em determinados esportes de resistência (ciclismo, esqui de campo e canoagem).

Os mesmos autores ${ }^{28}$ avaliaram também a anatomia, função e metabolismo cardíacos através de ressonância magnética e espectroscopia em ciclistas altamente treinados e em indivíduos controles. A massa ventricular esquerda e os volumes 
diastólicos finais, indexados pela superfície corpórea, foram significantemente maiores nos ciclistas, porém, a fração de ejeção ventricular esquerda, o índice cardíaco e o estresse parietal sistólico não diferiram dos controles. $O$ índice fosfocreatina/trifosfato de adenosina foi similar em ambos os grupos. Para os autores, esses achados indicam que a hipertrofia ventricular esquerda induzida pelo exercício físico é apenas adaptação fisiológica.

Medeiros et al..$^{30}$ demonstraram que os benefícios gerados pela prática regular, repetida e adequadamente intensificada do exercício físico resultam das interferências neuro-humorais que são despertadas durante a atividade física e que se sustentam durante parte, ou todo o período, dos intervalos das sessões de treinamento. Há dados consistentes indicando que da prática regular de exercício físico decorrem modificações favoráveis para o sistema circulatório relacionadas com a atividade simpática e parassimpática, com o sistema renina-angiotensina, com a redução da pós-carga, com a função endotelial e com as concentrações plasmáticas de aldosterona.

Diante do exposto com o presente estudo concluiu-se que o treinamento aquático foi mais eficiente na melhora dos níveis plasmáticos de lipídeos quando acompanhado por uma dieta adequada, confirmando que a associação de exercício físico com dieta equilibrada é essencial para obtenção de seus benefícios. Quando a HVE patológica já estava presente o treinamento aquático provavelmente diminuiu o depósito de colágeno intersticial, o que pode indicar uma melhora na função diastólica com consequente melhora da função sistólica.

Estabelecer intensidades e frequências adequadas de exercícios físicos é essencial para uma prescrição direcionada. Intervenções realmente efetivas, de baixo custo, são estratégias importantes para a saúde pública no Brasil, tanto no âmbito da promoção quanto da reabilitação à saúde, com impacto direto na qualidade de vida dos indivíduos.

\section{REFERÊNCIAS}

1. Mill JG, Vassallo DV. Hipertrofia cardíaca. Rev Bras Hipertens. 2001; 8(1): 18-29.

2. Oliveira E, Krieger J. Hipertrofia cardíaca e treinamento físico: aspectos moleculares. Hipertensão. 2002; 2(2): 73-8.

3. Franchini KG. Hipertrofia cardíaca: mecanismos moleculares. Rev Bras Hipertens. 2001; 8(1): 82-90.

4. Cressoni ES, Avanci LE, Braile DM, Cicogna AC, Gerez MAE, Martins AS, et al. Proteção miocárdica ao coração hipertrofiado: o eterno desafio. Rev Bras Cir Cardiovasc. 2008; 23(1): 18-29.

5. Graziosi P. Análise ecocardiográfica da estrutura e da função sistólica ventricular esquerda na hipertensão arterial. Hiperativo. 1998; 5(2): 23-31.

6. Rosenfeld ME, Polinsky P, Virmani R, Kauser K, Rubanyi G, Schwartz SM. Advanced atherosclerotic lesions in the innominate artery of the apoE knockout mouse. Arterioscler Thromb Vasc Biol. 2000; 20: 2587-92.

7. Calara F, Silvestre M, Casanada F, Yuan N, Napoli C, Palinski W. Spontaneous plaque rupture and secondary thrombosis in apolipoprotein E-deficient and LDL receptor-deficient mice. J Pathol. 2001; 195: 257-263.

8. Garcia JAD, Incerpi EK. Fatores e mecanismos envolvidos na hipertrofia ventricular esquerda e o papel anti-hipertrófico do óxido nítrico. Arq Bras Cardiol. 2008; 90(6): 45-51.

9. Evangelista FS, Brum PC, Krieger JE. Duration-controlled swimming exercise training induces cardiac hypertrophy in mice. Braz J Med Biol Res. 2003; 36(12): 25-30.

10. Friedewald WT, Levy RI, Fredrickson DS. Estimation of the concentration of low-density lipoprotein cholesterol in plasma, without the use of preparatory ultracentrifugation. Clin Chem. 1972; 18: 499-502. 
11. Armstrong AT, Binkley PF, Banker PB, Myerowitz D, Leier CV. Quantitative investigation of cardiomyocyte hypertrophy and myocardial fibrosis over 6 years after cardiac transplantation.J Am Coll Cardiol. 1998; 32: 704-10.

12. Halle M, Berg A, Garwers U, Baumstark MW, Knisel W, Keul J. et al. Influence of 4 Weeks' Intervention by Exercise and Diet on Low-Density Lipoprotein Subfractions in Obese Men With Type 2 Diabetes. Metabolism. 1999; 48(5): 641-44.

13. Pitanga FJG. Atividade física e lipoproteínas plasmáticas em adultos de ambos os sexos. Rev Bras Cien Mov. 2001; 9(4):25-31.

14. Kraus, WE, Houmard JA, Duscha BD, Knetzger KJ, Kulkarni KR, Slentz CA, et al. Effects of The Amount And Intensity of Exercise on Plasma Lipoproteins. N Engl J Med. 2002; 347: 1483-92.

15. Donovan G, Owen A, Bird SR, Kearney EM, Nevill AM, Jones DW. et al. Changes Cardiorespiratory fitness and coronary heart disease risk factors following $24 \mathrm{wk}$ of moderate or high intensity exercise of equal energy coast. J Appl Physiol. 2005; 98(6): 1619-25.

16. Leon AS, Sanchez OA. Response of blood lipids to exercise training alone or combined with dietary intervention. Med Sci Sports Exerc. 2001; 33(Suppl 6): S502-15.

17. Prado ES, Dantas EHM. Efeitos dos Exercícios Aeróbicos e de Força nas Lipoproteínas SHL, LDL e Lipoproteína. Arq Bras Cardiol. 2002; 79(4): 429-33.

18. Rique ABR, Soares EA, Meirelles CM. Nutrição e exercício na prevenção e controle das doenças cardiovasculares. Rev Bras Med Esp. 2002; 8(6): 89-96.

19. Bernardes D, Manzoni MSJ, DE Souza CP, Tenório N, Damaso AR. Efeitos da dieta hiperlipídica e do treinamento de natação sobre o metabolismo de recuperação ao exercício em ratos. Rev Bras Educ Fís Esp 2004; 18(2): 191-200.

20. Ghorayeb N, Batlouni M, Pinto IMF, Dioguard GS. Hipertrofia ventricular esquerda do atleta. Resposta adaptativa fisiológica do coração. Arq. Bras. Cardiol. 2005; 85(3): 21-30.

21. Pluim BM, Zwinderman AH, Laarse AV, Wall EED. The athlete's heart: a meta-analysis of cardiac structure and function. Circulation. 1999; 100: 336-44.

22. Natali AJ.Efeitos do exercício crônico sobre os miócitos cardíacos: uma revisão das adaptações mecânicas. Rev Bras Ciên Mov. 2004; 12(1): 91-6.

23. Medeiros A, Oliveira EM, Gianolla R, Casarini DE. Negrão CE, Brum PC. Swimming training increases cardiac vagal activity and induces cardiac hypertrophy in rats. Braz J Med Biol Res. 2004; 37(12): 1909-17.
Recebido 20/08/2013

Revisado 16/09/2013

$28 / 09 / 2013$

Aprovado 30/09/3013 